\title{
Scaling Data from Multiple Sources
}

\author{
Ted Enamorado* Gabriel López-Moctezuma ${ }^{\dagger} \quad$ Marc Ratkovic $^{\ddagger}$
}

July 8, 2019

\begin{abstract}
We introduce a method for scaling two data sets from different sources. The proposed method estimates a latent factor common to both datasets as well as an idiosyncratic factor unique to each. In addition, it offers a flexible modeling strategy which permits the scaled locations to be a function of covariates, and efficient implementation allows for inference through resampling. A simulation study shows that our proposed method improves over existing alternatives in capturing the variation common to both datasets, as well as the latent factors specific to each. We apply our proposed method to vote and speech data from the 112th U.S. Senate. We recover a shared subspace that aligns with a standard ideological dimension running from liberals to conservatives, while recovering the words most associated with each senator's location. In addition, we estimate a word-specific subspace that ranges from national security to budget concerns, and a vote-specific subspace with Tea Party senators on one extreme and senior committee leaders on the other.
\end{abstract}

${ }^{*}$ Assistant Professor, Department of Political Science, University of North Carolina at Chapel Hill, Chapel Hill NC 27514. Email: ted.enamorado@gmail.com, URL: http: / / www.tedenamorado.com

${ }^{\dagger}$ Assistant Professor, Division of the Humanities and Social Sciences, California Institute of Technology, Pasadena CA 91125. Email: glmoctezuma@caltech.edu, URL: http: / / glmoctezuma.com

${ }^{\ddagger}$ Assistant Professor, Department of Politics, Princeton University, Princeton NJ 08544. Phone: 608-658-9665, Email: ratkovicaprinceton. edu, URL: http: / / www . princeton. edu/ ratkovic 


\section{Introduction}

Increasingly, political scientists confront not just large amounts of data but different types of data. For example, political actors will often generate text data and vote data (e.g. Lauderdale and Clark, 2014); countries may have sets of qualitatively distinct attributes, such as political, social, and economic indicators (e.g. Coppedge et al., 2015); the same survey questions may be given to different groups of actors (e.g. Shor and McCarty, 2011); campaign contributions may flow from the same actors to both state and federal candidates (Bonica, 2014). In each case, the researcher must analyze data on different attributes for the same actors (say, tweets and votes from legislators, Barbera 2016), or the same attributes but on different actors (say, surveys given to both legislators and the mass public, Bafumi and Herron 2010).

The availability of multiple data sources allows researchers to exploit more information by combining datasets and recovering common traits across them. As it incorporates joint variation across multiple sources, estimating latent attributes from two datasets allows additional insights over an attribute obtained from just a single source of information.

As a first pass, the data from different sources may simply be pooled and scaled (Quinn, 2004; Hoff, 2007; Jackman and Trier, 2008; Murray et al., 2013). Pooling suffers, though, when one data set has much more information, swamping the information from the other set. Combining data from different sources creates even more subtle theoretical and empirical issues. Jessee (2016) illustrated the underlying problem rather elegantly. Using survey data for citizens and legislators, he showed that scaled locations can vary as the relative numbers of individuals from two samples are pooled and used to estimate ideological positions. The problem arises because the different groups give different weights to each question, and it generalizes to the problem of how to weight data coming from two different sources.

Existing approaches have addressed, but not quite solved, the issue of how to weight different types of 
data. For example, Kim, Londregan and Ratkovic (2018) develop a choice-theoretic model for combining words and votes, but a tuning parameter that balances the proportion of information coming from each source is not estimated within the model. The strength of this earlier work is grounding the estimates in a choice-theoretic model and estimating ideal points. As explained below, the method presented in this paper resolves the issue of how to optimally weight two data sources. In doing so, it eschews a formal choice-theoretic model, which allows it to extend to any two data sources, at the cost of returning scaled locations rather than estimated ideal points.

Authors like Hobbs (2017) combine information from multiple text sources using a version of canonical correlation analysis (e.g. Hastie, Tibshirani and Friedman., 2013, Sec. 3.7), a method closely related to ours. The method advanced by Hobbs (2017), though, is tailored to short bursts of speech and does not offer means of inference. Similarly, Weighted Multidimensional Scaling (WMDS) (Borg et al. (2013); Borg and Groenen (2005)) combines multiple dissimilarity matrices to recover a single underlying dimension (see also Jacoby, 1986, 2009). WMDS, though, returns only locations for the observations and not for the outcomes, i.e. votes or words, on which the observations are measured. This issue also plagues methods that must pre-select, rather than estimate, ideologically charged words (Groseclose and Milyo, 2005; Gentzkow and Shapiro, 2010; Martin and Yurukoglu, 2017).

We develop a general framework for combining data from multiple sources. The method, MultiDataset Multidimensional Scaling (MD2S) simultaneously scales two datasets, decomposing the data into three separate factors: one spanning a latent space common to both datasets, and two idiosyncratic subspaces - one per dataset. For example, combining votes and words on the same actors, MD2S estimates three latent scales. The first is a joint scale informed by both words and votes. The second is informed by words, but contains no information from votes. Likewise, the third is informed by votes, but not words.

We build off work in statistics and education focusing on recovering the correlation and shared factors 
across multiple surveys or exams (Tucker, 1958; Browne, 1979; Anderson, 1989; Klami, Virtanen and Kaski, 2013; Bach and Jordan, 2005; Gupta et al., 2011; Tipping and Bishop, 1999). This model, "InterBattery Factor Analysis," is precisely the model described above. We offer a likelihood-based method for optimally weighting the information coming from the two sources, allow the user to include covariates in estimating the scaled locations, and derive and implement an efficient algorithm for estimation.

The advantages of our proposed method are fourfold. First, we recover scaled locations for both observations (say, legislators) and features (say, text and votes). Estimating, for example, which words anchor a dimension's extremes greatly facilitates interpretation. Second, we allow for inference on the number of latent dimensions. Distinguishing a dimension that is signal from one that is noise is a perennial problem, often unaddressed, in the scaling literature. To this end, we implement a permutation test to distinguish a given dimension from noise. Our third advance is in terms of estimation. Building on insights first advanced in Aldrich and McKelvey (1977), we implement an efficient estimation routine that performs well when the number of attributes grows large, as with text data where the researcher has a document-term matrix with counts on thousands of $n$-grams for each speaker. Inference on the scaled locations for both shared and idiosyncratic subspaces is performed via bootstrapping. Finally, scaled locations are modeled as a function of covariates. This facilitates conducting inference on whether or how scaled locations relate to covariates of interest, giving a principled way to explore the estimated latent scales with substantive information.

We illustrate the method's use and efficacy through a simulation exercise and an empirical application. We show in the simulation study that MD2S recovers a shared and idiosyncratic dimensions more accurately than existing methods that combine multiple datasets, especially as the number of attributes grows large. We then apply it to roll call votes and floor speech in the US Senate, where there has been a long interest by congressional scholars on recovering legislators' latent scales. Our shared first dimension 
aligns with the standard ideological dimension running from liberals to conservatives recovered using only roll-call votes (e.g. Poole, 2005). By combining senators' votes and floor speech, we recover the selected words that differentiate Senators on this dimension, mostly on economic terms. Additionally, we further differentiate senators by recovering a word-specific subspace that ranges from national security to budget concerns, and a vote-specific subspace with Tea Party senators on one extreme and senior committee leaders on the other.

\section{Motivation and Use Cases}

To illustrate the basic problem and insight, consider the case where we observe two different streams of data, votes in a roll call matrix and word counts in a document-term matrix, that are observed on the same actors. As is common in text data, assume that we have many more words than votes. Were we to simply join the two datasets and estimate a single scale, it would be closer to the words-only scaling than the votes-only scaling. The words contain more information, but we are not interested in all of the word data. We are most interested in the word data that contribute to explaining the joint variation in both types of data. We could conduct multiple analyses after reweighting the matrix, to find a suitable balance between words and votes in the scaling procedure as in Kim, Londregan and Ratkovic (2018), but this sidesteps the problem of relative weighting rather than solving it.

MD2S solves this problem by returning three factors from the two datasets. The first is a joint factor, estimated to explain the largest amount of variance common to both datasets. The next two are idiosyncratic factors, unique to each data source and uncorrelated with the common factor: 


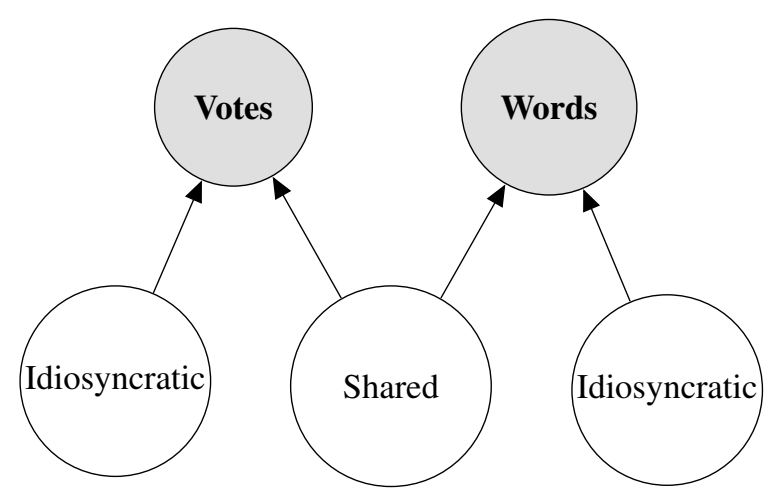

This model is the Inter-Battery Factor Analysis (IBFA) model of Tucker (1958). As noted above, MD2S builds on this model in several regards: estimating the number of latent dimensions, providing an efficient and effective estimation algorithm for a large number of attributes, and modeling the scaled locations with covariates.

Use Cases and Scope. If the data come from a single source, or the researcher is willing to ignore the problem of one data source overwhelming the other, then a standard principal components or factor analysis should be utilized. There may be several cases, though, where the researcher may wish to model the two different data sources. Our interest in this method was motivated by combining text and votes, where the sheer volume of the textual data may overwhelm the vote data. Beyond this particular case, the method's use fall into two broad categories: combining data sets and contrasting them.

Combining data sets may involve bringing auxiliary information to bear on a problem. For example, roll call votes are not informative in legislatures with strong party systems or in the presence of pressures for unanimous or lopsided voting, so words can be used to differentiate among members (for more, see Kim, Londregan and Ratkovic, 2018; Kellerman, 2012). Relatedly, one data source may not have sufficient signal to generate a reliable scale, so a second data source can help leverage the first (e.g., Hobbs, 2017). Combining the two sources offers an additional benefit. Placing words and actors in the same space, as in our applied example, allows the researcher to use the selected features to characterize the substantive 
meaning of each dimension.

Bridging across different actors is another form of combining data. Jessee (2016) highlighted the problem of weighting data from different sources when bridging across different sets of respondents (e.g., Lewis and Tausanovitch, 2015; Tausanovitch and Warshaw, 2013; Shor and McCarty, 2011; Bafumi and Herron, 2010). If the two groups have different item discrimination parameters, simply pooling the two sets generates ambiguity in their ideal point estimates. The estimates will vary based on either the amount of information or based on the number of respondents, in the two sets. ${ }^{1}$

A third instance for combining data sets comes when constructing indices. Consider the impressive set of measures for cross-national political, civic, and institutional comparison assembled by Coppedge et al. (2015). Generating an index by aggregating from finer to coarser measures requires a method that is not sensitive to the number of items at each level.

A second use of the method is for contrasting the two information sources. This approach differs from methods that only uncover a single scale; we discuss these methods in more detail below. MD2S offers the ability to isolate a set of factors based off whether they are informing both data sets, or exclusively one or the other. With data on word usage on the same individuals before and after an event, three sets of factors can be recovered: a factor common to both before and after, one unique to before and uninformed by after the event, and one unique to after the event and not informed by information before the event. Examples of this analysis involve contrasting Twitter data (Barbera, 2016), transcripts of Federal Open Market Committee Meetings before and after a transparency shock (Hansen, McMahon and Prat, 2018), or Weibo microblogs before or after censorship (Hobbs and Roberts, 2018). Our method offers a structured way of separating out a common factor, allowing the researcher to estimate how latent factors vary across the two datasets.

\footnotetext{
${ }^{1}$ Section B.1 in the Appendix presents and discusses a simulation-based example of how our method can be used for bridging.
} 


\section{The Proposed Method}

For each observation, we observe two vectors of outcomes, $Y_{(1) i}^{*}$ and $Y_{(2) i}^{*}$ with $i \in\{1,2, \ldots, N\}$. To ease notation, we will use the index $m$ to denote either 1 or 2 , so $Y_{(m) i}^{*}$ is the generic notation for either $Y_{(1) i}^{*}$ or $Y_{(2) i}^{*}$. We assume $Y_{(m) i}^{*}$ is of length $K_{m}$, where $K_{1}$ may not equal $K_{2}$, and we use $Y_{(m) j}$ to denote the $N$ values of the $j^{\text {th }}$ feature from data source $m$. For example, $Y_{(1) i}^{*}$ may be a vector of $K_{1}$ word counts uttered by legislator $i, Y_{(2) i}^{*}$ may be a set of $K_{2}$ observed roll call votes for the same legislator, and $Y_{(1) j}$ is the vector of $N$ counts for a word $j \in\left\{1,2, \ldots, K_{1}\right\}$ while $Y_{(2) j}$ are the $N$ outcomes for vote $j \in\left\{1,2, \ldots, K_{2}\right\}$.

We denote the outcome data matrices as:

$$
Y_{(1)}^{*}=\left[\begin{array}{c}
Y_{(1) 1}^{* \top} \\
Y_{(1) 2}^{* \top} \\
\cdots \\
Y_{(1) N}^{* \top}
\end{array}\right] ; \quad Y_{(2)}^{*}=\left[\begin{array}{c}
Y_{(2) 1}^{* \top} \\
Y_{(2) 2}^{* \top} \\
\\
\cdots \\
Y_{(2) N}^{* \top}
\end{array}\right] .
$$

with individual outcomes in rows. We assume that each matrix $Y_{(m)}^{*}$ is on a common scale. This may be due to a natural scale, such as binary vote data, columns may be normed to have sample standard deviation one, or some other method may be used to place all columns of $Y_{(m)}^{*}$ on a common scale (e.g., Quinn, 2004; Hoff, 2007; Murray et al., 2013). The important point for our method is that all columns of $Y_{(m)}^{*}$ be on a common interval scale. While each matrix must be on a common scale, the two separate matrices may be on different scales. For example, $Y_{(1)}^{*}$ may contain roll call votes and $Y_{(2)}^{*}$ word counts. 


\subsection{The Model}

In practice, as the intercept is rarely of interest, we pre-process the matrices by double-centering them, so that the row-mean, column-mean, and grand mean is zero. We denote the double-centered matrices as $Y_{(m)} \cdot{ }^{2}$ Thus, we model $Y_{(1)}$ and $Y_{(2)}$ in terms of their latent factors as ${ }^{3}$

$$
\begin{aligned}
& Y_{(1)}=Z_{S} L_{(1)} W_{(1)}^{\top}+Z_{(1)} D_{(1)} B_{(1)}^{\top}+\Omega_{(1)} \\
& Y_{(2)}=Z_{S} L_{(2)} W_{(2)}^{\top}+Z_{(2)} D_{(2)} B_{(2)}^{\top}+\Omega_{(2)} .
\end{aligned}
$$

We will refer to the $N \times Q_{S}$ matrix $Z_{S}$ as the shared subspace and the $N \times Q_{(m)}$ matrix $Z_{(m)}$ as the idiosyncratic subspace. $Z_{S}$ contains latent locations on the shared subspace in columns for each of the $Q_{S}$ dimensions. Similarly, each column of $Z_{(m)}$ contains the latent locations in the idiosyncratic subspace for $Q_{(m)}$ latent dimensions. $L_{(m)}$ is a $Q_{S} \times Q_{S}$ nonnegative, diagonal matrices of loadings for the shared subspace. We assume that the two matrices $L_{(1)}$ and $L_{(2)}$ are proportional, so any difference between them is attributable to the relative scales across data sources $Y_{(m)} . W_{(m)}$ is a $Q_{(m)} \times Q_{(m)}$ matrix of factors for the shared subspace for dataset $Y_{(m)} . D_{(m)}$ is a $Q_{(m)} \times Q_{(m)}$ diagonal matrix of loadings for the idiosyncratic subspace, and $B_{(m)}$ is the $K_{(m)} \times Q_{(m)}$ of factors for the idiosyncratic subspace. The matrix $\Omega_{(m)}$ is a matrix of mean-zero, independent, equivariant noise.

We have modeled each observed data matrix $Y_{(m)}$ in terms of a shared subspace $Z_{S}$ and individual subspaces $Z_{(m)}$. The researcher may believe, though, that the estimated scaled locations may vary systematically with some set of known covariates available for the $N$ observations in the data. As in Roberts

\footnotetext{
${ }^{2}$ The maximum likelihood estimates for the intercept terms are the sample analogs (Tipping and Bishop 1999), i.e. the double-centering matrix with each element the row-mean plus the column-mean, less the grand mean. See Poole and Rosenthal 1997 for a discussion of double-centering.

${ }^{3}$ We chose notation consistent with Murphy (2012) and Klami, Virtanen and Kaski (2013). We denote all observed outcomes as $Y_{(m)}$ instead of $Y$ and $X$.
} 
et al. (2014), we allow the scaled locations to take the following form:

$$
\begin{aligned}
Z_{S} & =X_{S} \beta_{S}+\Omega_{Z_{S}} \\
Z_{(m)} & =X_{(m)} \beta_{(m)}+\Omega_{Z_{(m)}}, \text { for } m \in\{1,2\}
\end{aligned}
$$

The covariates $X_{S}, X_{(m)}$ structure the systematic factors of $Z_{S}, Z_{(m)}$, respectively.

We make five assumptions for identifying the model (for a discussion of identification, see Tipping and Bishop, 1999, Appendix A.1), where the assumptions hold for $m \in\{1,2\}$ :

$$
\begin{aligned}
& Z_{S}^{\top} Z_{S}=W_{(m)}^{\top} W_{(m)}=I_{Q_{S}} \\
& Z_{(m)}^{\top} Z_{(m)}=B_{(m)}^{\top} B_{(m)}=I_{Q_{(m)}} \\
& Z_{(m)}^{\top} Z_{S}=\mathbf{0}_{Q_{(m)} \times Q_{S}} \\
& L_{(1)} \propto L_{(2)} \\
& L_{(m)}, D_{(m)} \text { are diagonal with non-negative entries }
\end{aligned}
$$

Assumptions (6) - (7) state that, within a given subspace, the latent scalings and factors are uncorrelated and length one. Assumption (8) states that the common subspace spanned by $Z_{S}$ is not correlated with the idiosyncratic scalings. This assumption allows us to differentiate the shared subspace from each idiosyncratic subspace. Assumption (9) requires the variation in loadings for the shared subspace to be explained by the relative scales across data sources. Assumption (10) identifies the particular rotation that we estimate. Specifically, we are assuming that the factors $W_{(m)}$ and $B_{(m)}$ are numerically equal to singular decompositions of the shared and idiosyncratic subspaces of $Y_{(m)}$, respectively. ${ }^{4}$ Note that we

\footnotetext{
${ }^{4}$ See (Tipping and Bishop, 1999) and our discussion below for more.
} 
only identify the latent factors $Z_{S}, Z_{(m)}, W_{S}$ and $B_{(m)}$ up to sign. ${ }^{5}$ We follow convention and assume the elements of $L$ and $D_{(m)}$ are nonnegative and arranged in decreasing order. We discuss relaxations of these assumptions in Section 3.5.

\subsection{A Probabilistic Framework}

We next embed our factor model in a probabilistic framework, where we recover maximum likelihood estimates of the factors. The probabilistic MD2S model can be written as

$$
Y_{(m)} \mid W_{(m)}, B_{(m)}, L_{(m)}, D_{(m)} \sim \mathcal{N}\left(Z_{S} L_{(m)} W_{(m)}+Z_{(m)} D_{(m)} B_{(m)}, \sigma_{(m)}^{2} I_{N}\right)
$$

This model is an extension of the Probabilistic Principal Components model of Tipping and Bishop (1999); see also Bach and Jordan (2005). We differ from these models as we are most interested in the actors' spatial locations $\left(Z_{S}, Z_{(m)}\right)$, so we treat the weights $W_{(m)}$ and $B_{(m)}$ as random and the spatial locations as fixed (see also Aldrich and McKelvey, 1977, p. 117). We maintain the assumption that the errors are of equal variance, and therefore do not vary systematically across individuals or features.

Marginalizing over $W_{(m)}$ and $B_{(m)}$, gives the unconditional densities for $Y_{(m)}$ as

$$
Y_{(m)} \sim \mathcal{N}\left(0_{N}, C_{m}\right)
$$

where $C_{m}=Z_{S} L_{(m)}^{2} Z_{S}^{\top}+Z_{(m)} D_{(m)}^{2} Z_{(m)}^{\top}+\sigma_{(m)}^{2} I_{N}$, for $m=1,2$.

\footnotetext{
${ }^{5}$ This means that the data cannot differentiate between a model with estimates $\left\{Z_{S}, Z_{(m)}, W_{S}, B_{(m)}\right\}$ and $\left\{-Z_{S},-Z_{(m)},-W_{S},-B_{(m)}\right\}$.
} 
The data log-likelihood as a function of $\left(Z_{S}, Z_{(1)}, Z_{(2)}, L_{(1)}, L_{(2)}, D_{(1)}, D_{(2)}\right)$ can be written as

$$
\begin{aligned}
& l\left(Z_{S}, Z_{(1)}, Z_{(2)}, L_{(1)}, L_{(2)}, D_{(1)}, D_{(2)} \mid Y_{(1)}, Y_{(2)}\right)= \\
& -\frac{1}{2}\left\{N\left(K_{1}+K_{2}\right) \log (2 \pi)-K_{1} \log \left(\left|C_{1}\right|\right)-K_{2} \log \left(\left|C_{2}\right|\right)-\operatorname{tr}\left(Y_{(1)} Y_{(1)}^{\top} C_{1}^{-1}+Y_{(2)} Y_{(2)}^{\top} C_{2}^{-1}\right)\right\}
\end{aligned}
$$

We derive an expression and results for the maximum likelihood estimates in Appendix A.

\subsection{Implementation}

In the single dataset setting, Tipping and Bishop (1999) show that the maximum likelihood estimates for each factor are principal components of the data. We extend the result to the MD2S model. Doing so allows for an efficient estimation strategy, whereby we can estimate $Z_{S}, Z_{(1)}$, and $Z_{(2)}$ directly using an

iterative algorithm, then recover the remaining estimates, $\hat{W}_{(m)}, \hat{B}_{(m)}, \hat{L}_{(m)}, \hat{D}_{(m)}$, afterwards. We prove the validity of this strategy in the following proposition:

PROPOSITION 1 The maximum likelihood estimates for the shared and idiosyncratic subspaces can be written as singular vectors of functions of the data. Specifically:

1. The maximum likelihood estimates for $Z_{(m)}$ are proportional to principal components of $Y_{(m)}^{\top} M\left(Z_{S}\right)$ for $m=1,2$.

2. Denote $Z_{S \mid m}$ as the first $L_{S}$ principal components of $Y_{(m)}^{\top} M\left(Z_{(m)}\right)$. Then,

(a) $Z_{S} \propto Z_{S \mid 1} w_{1}+Z_{S \mid 2} w_{2}$ with $w_{1}+w_{2}=1 ; w_{1}, w_{2}>0$ and

(b) $Z_{S}$ is selected to maximize $\operatorname{tr}\left(Z_{S}^{\top} Y_{(1)} Y_{(1)}^{\top} Y_{(2)} Y_{(2)}^{\top} Z_{S}\right)$.

where $M(A)$ is the annihilator matrix for matrix $A: I-A\left(A^{\top} A\right)^{-1} A^{\top}$ with $\left(A^{\top} A\right)^{-1}$ denoting the generalized inverse of $\left(A^{\top} A\right)$ and I is the commensurate identity matrix. 


\section{Proof. See Appendix A.}

The proposition leads directly to our estimation strategy. ${ }^{6}$ Our algorithm estimates the MD2S model using an iterative procedure that updates the estimate of each subspace one at a time, enforcing the constraints in Equations 6-10 along the way. That is, for every iteration until convergence, the estimation proceeds in two steps. Given the previous iteration estimate of the shared space $Z_{S}$, we update $\hat{Z}_{(1)}$ and then $\hat{Z}_{(2)}$. Second, we partial the idiosyncratic spaces out to update $\hat{Z}_{S}$. After convergence in each subspace, we update our estimates of the remaining parameters.

Note that our algorithm allows the computational advantage of having to invert square matrices of whichever size is smaller, $N \times N$ or $K_{(m)} \times K_{(m)} \cdot{ }^{7}$ For example, in the main empirical application below we observe 100 voting members, 486 votes, but 2,532 words. Our algorithm is fit through inverting matrices of size $100 \times 100$ instead of $486 \times 486$ or $2,532 \times 2,532$, which gives us sizable computational gains. One advantage of our algorithm is that, at each step, it recovers estimates of the data, $\widehat{Y}_{(1)}$ and $\widehat{Y}_{(2)}$, conditional on current estimates of shared and idiosyncratic subspaces. Thus, all the information at hand is used in estimation.

\subsection{Uncertainty}

We estimate uncertainty for two parts of the MD2S model: the scaled locations and the number of dimensions. For the scaled locations, we rely on the bootstrapping methodology introduced by Jacoby and Armstrong II (2014). Let $\left\{\tilde{Y}_{(1), b}^{*}, \tilde{Y}_{(2), b}^{*}\right\}$ denote two $b^{\text {th }}$ bootstrapped sample, with $b \in\{1,2, \ldots, B\}$, where $B$ is some large number, such as 1,000 . The bootstrapped sample is generated by fixing the number of rows and sampling $K_{(m)}$ columns for each matrix, with replacement. Uncertainty due to sampling error can be estimated through fitting MD2S to these bootstrapped estimates.

\footnotetext{
${ }^{6}$ See the Supplemental Appendix A for details.

${ }^{7}$ See Aldrich and McKelvey (1977, p. 117) and Tipping and Bishop (1999, Appendix B) for similar insights.
} 
We present a statistical method for estimating the number of dimensions while acknowledging that the first empirical consideration should be substantive interpretability of the estimated subspaces. We recommend separating signal from noise dimensions through the use of a permutation test (e.g. Keele, McConnaughy and White, 2012). A permutation test requires estimating the density of a test statistic on a set of datasets permuted such that under the null hypothesis, there is in-truth no signal in the data, and then the observed value is compared to this simulated null distribution. We are not the first to use a permutation test to separate an estimated scale from noise (see e.g., Mair, Borg and Rusch 2016 and references therein). However, these authors only compare the estimated weight on each dimension to the mean under the simulated null, rather than estimate a $p$-value (Figure 1 in Mair, Borg and Rusch 2016).

For the permutation test, we assume that there is no structure in the data, so the subspace loadings are all zero. Formally,

$$
\begin{array}{r}
\mathcal{H}_{(m) L, q}^{0}: L_{q}=0 ; \\
\mathcal{H}_{(m) D, q}^{0}: D_{(m) q}=0
\end{array}
$$

for all $(m, q)$, where $L_{q}=L_{(1) ; q}$ and $q$ indexes a given dimension. Under these hypotheses, the observed data is pure noise with no systematic structure, i.e. $Y_{(m)}=\Omega_{(m)}$.

Under these null hypotheses, any permutation of the data is equally likely. We permute the data, estimate dimension weights, and then compare the statistic under the observed data to the statistic under the null distribution. To the extent that the statistic is an outlier under the null hypothesis, we can argue that the null hypothesis is not accurate and there is, in fact, some systematic relationship in the data.

Specifically, we permute the data such that within each column of $Y_{(m)}$, the rows are shuffled. In this case, in truth, there is no systematic relationship in the permuted data. Denote the $r^{\text {th }}$ permuted dataset 
out of $R$ total as $\tilde{Y}_{(m)}^{r}$, with $R$ some large number, say 1000 . For each permuted dataset, we calculate the dimension weights, $\widehat{L}_{q}^{r}$ and $\widehat{D}_{(m) ; q}^{r}$. These values are then compared to the estimated values on the non-permuted data, $\widehat{L}_{q}$ and $\widehat{D}_{(m) ; q}$.

Under this formulation, a $p$-value for dimension $q$ in the shared subspace or idiosyncratic subspace can be estimated as

$$
\hat{p}_{S ; q}=\frac{\sum_{r=1}^{R} \mathbf{1}\left(\widehat{L}_{q}^{r} \leqslant \widehat{L}_{q}\right)}{R} ; \hat{p}_{(m) ; q}=\frac{\sum_{r=1}^{R} \mathbf{1}\left(\widehat{D}_{(m), q}^{r} \leqslant \widehat{D}_{(m) ; q}\right)}{R} ;
$$

We adapt the test to our model by noting that the tests are not independent. The dimensions are estimated in order of decreasing loadings, such that more explanatory dimensions are estimated before less explanatory ones. Therefore, we take as our estimated dimensionality the first $d$ dimensions such that each dimension has an estimated $p$-value below a given threshold. In our empirical applications, we calculate the estimated dimensionality $\widehat{d}=q$ as the largest $q$ such that dimensions 1 to $q$ have estimated $p$ values below 0.1 . However, once the permuted $p$-value is estimated, this threshold can be be manipulated to assess the sensitivity of the estimated number of dimensions. ${ }^{8}$

\subsection{Extensions and Discussion of Method}

By using a probabilistic model, we are able to augment the model in order to extend the MD2S model to a large class of problems. For example, we can turn the model into a quadratic utility model through utilizing the latent normal representation of a probit model (Clinton, Jackman and Rivers, 2004; Albert and Chib, 1993; Hare et al., 2015; Jackman and Trier, 2008), leaving it commensurate with popular random utility models (e.g. Ladha, 1991). We can also utilize scale- and location-mixtures of normals to accommodate ordinal and count data, as in Goplerud (2019); Albert and Chib (1993). In this framework, our probabilistic

\footnotetext{
${ }^{8}$ Formally, $\widehat{d}_{S}=\operatorname{argmin}_{q}\left\{q: \hat{p}_{S ; q}>0.1\right\}-1 ; \quad \widehat{d}_{(m)}=\operatorname{argmin}_{q}\left\{q: \widehat{p}_{(m) ; q}>0.1\right\}-1$
} 
model is the "M"-step of an EM routine, with the "E"-step as an adjustment to the observed data. ${ }^{9}$ Our concern here is not with accommodating a particular class of data, such as binary, ordinal, or count data, but instead to develop a framework for integrating multiple sources in a single coherent fashion.

Other possible extensions can be integrated into the MD2S framework. For example, rather than identifying the factors through orthogonality conditions, the researcher could instead allow for correlated factors and instead identify them with a prior; see Klami, Virtanen and Kaski (2013); Gupta et al. (2011) for recent work. Placing a prior could shrink elements of the factor, returning a set of correlated factors that may be easier to interpret, particularly in high-dimensional settings (see, e.g. Rockova and George, 2016, for work in a factor model). In addition, a sparsity prior on the dimension weights could be used to select the number of underlying dimensions (e.g. Kim, Londregan and Ratkovic, 2018; Hahn, Carvalho and Scott, 2012). Using orthogonality conditions guarantee identification and simplify several of the derivations in our estimation algorithm (see Appendix A), but placing more structure on the factors can be incorporated into our model.

We have also assumed that all of the columns in $Y_{(m)}$ are on the same scale. If an analysis requires combining data on different scales, say a combination of continuous and categorical outcomes, we have two suggestions. First, if all of the data is continuous and approximately normal, each column may be converted to a $z$-scale by subtracting off the mean and dividing by the sample standard deviation. Recent literature has also suggested placing data on the same scale through an inverse $z$-transformation of the empirical distribution function,

$$
Y_{(m) ; i j}^{z}=\Phi^{-1}\left(\frac{1}{N+1} \sum_{i^{\prime}=1}^{N} \mathbf{1}\left(Y_{i^{\prime} j} \leqslant Y_{i j}\right)\right)
$$

\footnotetext{
${ }^{9}$ For example, in a latent probit model, this step involves adding to the fitted values the mean of a normal covariate truncated at 0 and centered at the fitted value, with support above zero for observed values of " 1 " or below zero for observed values of "0," and support over the whole line for missing values. See Clinton, Jackman and Rivers (2004); Albert and Chib (1993).
} 
where $\Phi(\cdot)$ is the normal distribution function. For more on this and other methods, see Quinn (2004); Hoff (2007); Murray et al. (2013).

The probabilistic PCA model allows us to recover point estimates even when there are more features than observations, causing existing common factor analytic implementations to fail due to a rank deficiency. This data structure is unavoidable in text data, where word features may greatly outnumber units of observation. A second approach, Weighted Multidimensional Scaling (WMDS) (Borg et al., 2013; Borg and Groenen, 2005), returns a common index across several data sets, with a measure of how much information each dataset contributes to the common index. MD2S optimally combines the two datasets to extract a common factor, as in WMDS, as well as idiosyncratic factors, allowing the researcher to estimate the location of features along each estimated scale. Doing so greatly aids interpretation, since we can use both the observations (e.g., legislators) and their features (e.g.,words) to summarize the dimensions.

Lastly, we wish to qualify how the $p$-values should be incorporated into the process of interpretation. Our permutation test offers a precise, but incomplete, measure of uncertainty; see Mair, Borg and Rusch (2016, esp. 778-779) for recent work on the topic. ${ }^{10}$ We advocate three different criteria for ascertaining whether an uncovered dimension is systematic. First is the $p$-value. If a dimension is not easily distinguished from noise, it should not be favored. This, of course, is necessary but not sufficient. The second criterion we recommend is substantive significance, namely the proportion of the observed variance explained by the method. The third criterion is whether the dimension has face validity. Every positive $p$-value threshold leaves open the possibility of recovering a noise dimension, so the particular threshold should be selected based off the researcher's tolerance of false positives. We follow convention and implement a threshold of 0.1 below, but do so while emphasizing that the final elements of evaluating the recovered dimensions rely crucially on substantive understanding. If the three criteria we list lead to dif-

\footnotetext{
${ }^{10}$ We note that these authors advocate comparing the mean dimension weight under the null to the observed value rather than calculating a proper $p$-value; see, e.g., Figure 1 in Mair, Borg and Rusch (2016).
} 
fering conclusions on whether a dimension is systematic, there is no perfect answer: we advocate that the researcher carefully and transparently adjudicate amongst the criteria, bringing theory and existing results into the process.

\section{Simulation Study}

In order to assess the proposed method, we conduct a simulation study which tests MD2S across two different elements: first, its ability to identify common and idiosyncratic factors, as well as its ability to distinguish systematic dimensions from noise.

\subsection{Simulation Setup}

The observed data consist of matrices $Y_{(1)}$ and $Y_{(2)}$ with $N$ rows and $K_{1}$ and $K_{2}$ columns respectively. $N$ is varied along $\{20,50,100\}$ and $K_{2}$ along $\{20,100,250,500,1000,2500,5000\} . K_{1}$ is held at 40 . The data are generated as

$$
\begin{aligned}
& Y_{(1)}=2 Z_{S 1} W_{(1) 1}^{\top}+Z_{S 2} W_{(1) 2}^{\top}+4 Z_{(1) 1} B_{(1) 1}^{\top}+2 Z_{(1) 2} B_{(1) 2}^{\top}+\Omega_{(1)} \\
& Y_{(2)}=2 Z_{S 1} W_{(2) 1}^{\top}+Z_{S 2} W_{(2) 2}^{\top}+4 Z_{(2) 1} B_{(2) 1}^{\top}+2 Z_{(2) 2} B_{(2) 2}^{\top}+2 Z_{(2) 3} B_{(2) 3}^{\top}+\Omega_{(2)}
\end{aligned}
$$

There are two shared dimensions, one twice the size of the other. The matrix $Y_{(m)}$ has two idiosyncratic dimensions and $Y_{(2)}$ has three. All systematic factors $Z . ., W_{(m)}$., and $B_{(m)}$. are drawn from a standard normal. The error matrices $\Omega_{(m)}$ are scaled such that the systematic component has twice the standard

error of the random component, i.e. the true $R^{2}$ is $(2 /(2+1))^{2}=4 / 9 \approx 0.44$. All simulations were run 1,000 times.

We designed this simulation with two goals in mind. First, we wanted the common factor in $Z_{S}$ to not be the largest systematic factor of $Y_{(1)}$ and $Y_{(2)}$. Uncovering the common factor involves avoiding the 
idiosyncratic factors. Second, we wanted to have more variables than observations in one of the matrices. We did so to mimic text data, where we have more terms than observations and regular factor analysis is computational infeasible.

We compare our proposed algorithm to two additional methods that are able to recover a shared scale from multiple datasets. First, we use a variational approximation of the Bayesian Inter-Battery Factor Analysis (V-BIBFA) model of Klami, Virtanen and Kaski (2013). The data generating process behind V-BIBFA is the same as ours, which is based on a linear latent variable model. In contrast to MD2S, V-BIBFA targets the factors or linear projections $W_{(m)}$ and $B_{(m)}$ instead of the latent factors $Z_{S}$ and $Z_{(m)}$. This is done by placing a sparse prior over the linear projections in order to separate a shared linear mapping $W_{(m)}$ from a specific one for each dataset $m, B_{(m)}$. Given an estimated posterior distribution of factors, scaled locations can be recovered from a normal posterior.

We also compare MD2S to another scaling approach, weighted multidimensional scaling or "individual differences scaling" (INDSCAL) as implemented in the $\mathbf{R}$ library smacof. Instead of focusing on the scaling of two matrices of size $N$ by $K_{1}$ and $N$ by $K_{2}$ as done by MD2S, INDSCAL recovers a shared scale from two matrices of dissimilarities of size $N$ by $N$ instead. First, a scale is recovered for each individual dataset and a matrix of weights is estimated to map this individual scales into a shared subspace. ${ }^{11}$ We use the Manhattan distance (L1 norm) as the measure of dissimilarity between the rows of $Y_{(1)}$ and $Y_{(2)}$ to reduce them to square matrices of size $N$ by $N$. In contrast to MD2S, INDSCAL does not directly return shared and idiosyncratic variation. In order to extract data-specific scales orthogonal to the shared subspace, we first estimate the shared latent dimension with the INDSCAL procedure. Next, with a linear mapping we partial this scale out from the $K_{m}$ outcomes in each original data matrix, $Y_{(m)}^{*}$. Finally, each partialed out dataset is transformed into a squared dissimilarity matrix and scaled via metric

\footnotetext{
${ }^{11}$ We thank an anonymous reviewer for suggesting this comparison.
} 


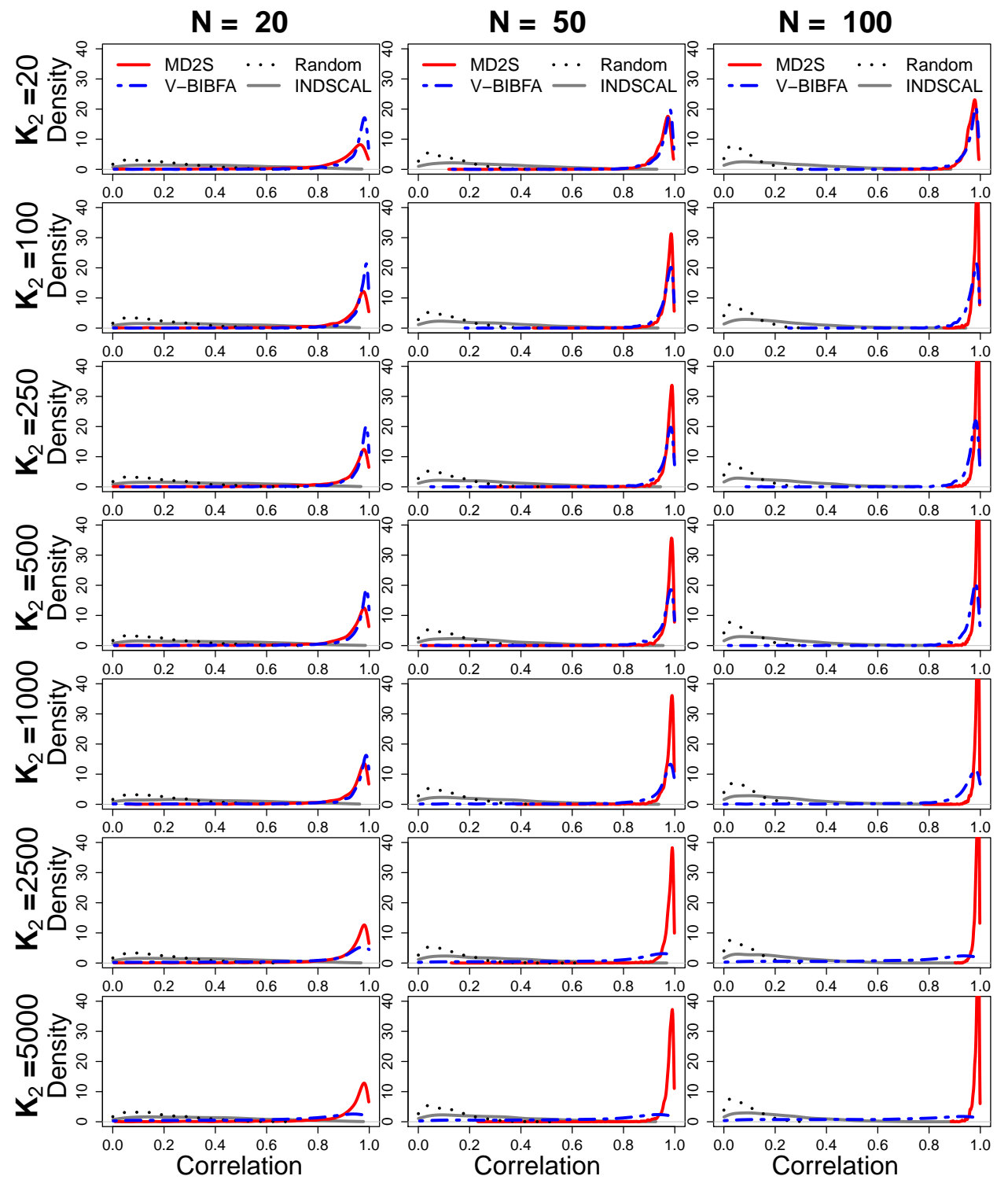

Figure 1: Correlation between Common Subspace $\left(Z_{S}\right)$ and its Estimate, by Method. Sample size is in columns $(N \in\{20,50,100\})$ and $K_{2} \in\{20,100, \ldots, 5000\}$ is in rows. The $x$-axis ranges from 0 to 1 and measures the correlation between the true and estimated values. The proposed method, MD2S, is compared to V-BIBFA and INDSCAL, as described in the text. All methods improve in $N$, but MD2S alone also improves in $K_{2}$. The remaining methods deteriorate in $K_{2}$. Across settings, either MD2S or V-BIBFA performs the best.

multidimensional scaling, as implemented in the $\mathbf{R}$ library smacof. 


\subsection{Results}

Our primary interest is in recovering a scaling informed by both sets of datasets. Figure 1 presents the results comparing estimates of the shared subspace and the true shared subspace. The figure is organized with sample size in columns $(N \in\{20,50,100\})$ and the number of outcomes in $Y_{(2)}, K_{2} \in$ $\{20,100, \ldots, 5000\}$ in rows. The $x$-axis ranges from 0 to 1 and measures the correlation between the true and estimated values. The $y$-axis is a density scale. The methods presented include Multi-Dataset Multidimensional Scaling (MD2S), the proposed method; the variational Bayesian implementation of Klami, Virtanen and Kaski (2013) (V-BIBFA); the individual differences scaling (INDSCAL); and an estimate that is pure normal noise (Random).

Looking across the columns of figure 1, each method benefits from an increase in sample size and is clearly differentiable from random noise. We see that, across settings, either MD2S or V-BIBFA performs the best in recovering the true shared subspace. Particularly when $N=20$ and $K_{2}$ is less than 1000 , V-BIBFA returns the best estimates of $Z_{S}$; the remainder of the time, MD2S performs the best. Note that, as $K_{2}$ increases, the estimates in V-BIBFA deteriorate in $K_{2}$ while MD2S improves. This is evidence that, with large outcome data matrices, such as the ones generated by text data, our iterative algorithm is able to recover a latent shared subspace that is closer to the true data generating process than available alternatives. The solid gray line shows that INDSCAL regularly outperforms random noise, but performs notably worse than MD2S and V-BIBFA.

Figure 2 contains the same set of results as Figure 1, but for the idiosyncratic subspaces $Z_{(1)}$ from $Y_{(1)}\left(\right.$ left), and $Z_{(2)}$ from $Y_{(2)}$ (right). Consider the left-side panel. The number of features in $Y_{(1)}, K_{1}$, is fixed across simulations, only $K_{2}$ is changing. Looking down rows, we see that the MD2S and V-BIBFA results for $Z_{(1)}$ change little with changes in $Y_{(2)}$. This is desirable: since $Z_{(1)}$ is idiosyncratic to $Y_{(1)}$, we 

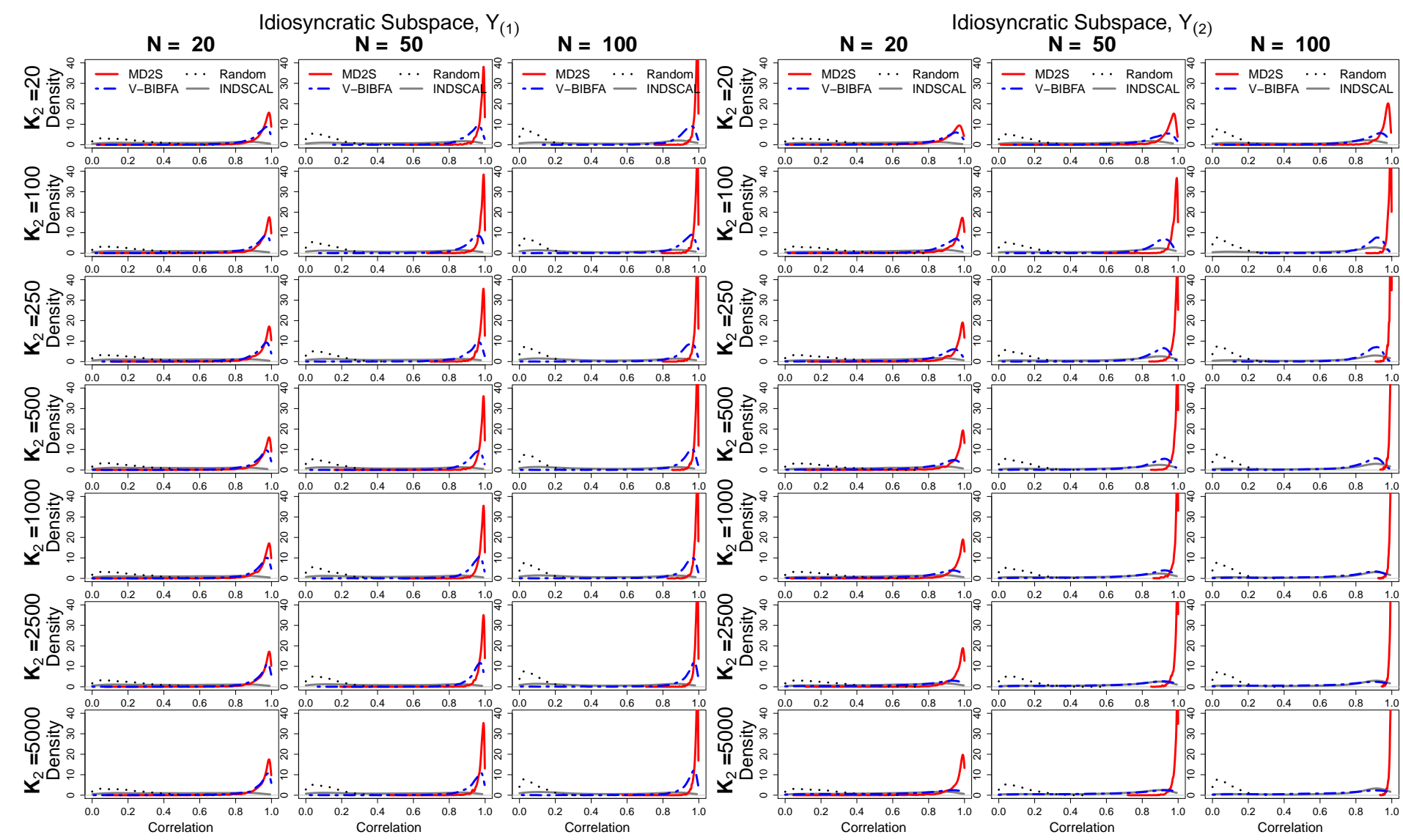

Figure 2: Correlation between Idiosyncratic Locations for $Y_{(m)}$ and their Estimates, by Method. The left and right figures are structured identically to Figure 1. Consider the left figure. Looking down rows, MD2S estimates for $Z_{(1)}$ change little with changes in $Y_{(2)}$. Other methods are similarly stable. Looking across columns, as $N$ increases, MD2S and V-BIBFA perform the best and nearly equivalently, though MD2S seems to have a slight edge. Now, consider the right-hand figure. Looking across columns, all methods improve as $N$ increases. As both $N$ and $K_{2}$ increase, the performance of V-BIBFA deteriorates, while MD2S improves. For $N \in\{50,100\}$ and $K>100$, MD2S is outperforming all other methods in recovering $Z_{(2)}$. When $K_{2} \geqslant 1000$ and $N \geqslant 50, \mathrm{MD} 2 \mathrm{~S}$ recovers $Z_{(2)}$ near exactly, and quite a bit better than all other methods.

do not want changes in $Y_{(2)}$ to impact its estimate. Overall, INDSCAL's performance is relatively not the best, with an average correlation with $Z_{(1)}$ of just 0.33 . Looking across columns, as $N$ increases, we see that MD2S and V-BIBFA perform better, although the correlation between MD2S estimates and the true subspace $Z_{(1)}$ is significantly more precise than for the V-BIBFA results.

Next, consider the right-hand panel. Looking across columns, again, we see all methods except INDSCAL improving as $N$ increases. However, similar to the case of the shared subspace, as $K_{2}$ increases, 
the performance of V-BIBFA deteriorates, while MD2S improves. In fact, MD2S outperforms V-BIBFA in recovering $Z_{(2)}$ across all configurations. Moreover, when $K_{2} \geqslant 500$ and $N \geqslant 50$, MD2S recovers $Z_{(2)}$ near exactly and better than all other methods.

Beyond scaling, bridging is a particularly important potential application of our proposed model, and worthy of more complete study in its own right. To this end, we present some preliminary simulation results on bridging in Supplemental Appendix B. We find that encouraging evidence that MD2S performs well at this task, but we are providing a more comprehensive discussion and exploration of this topic in a separate manuscript.

\subsection{Estimating Dimensions}

We next illustrate the proposed method's ability to separate systematic from noise dimensions through the use of the permutation test presented in section 3.4.

Figure 3 presents the results of the permutation test described in section 3.4 , for which five dimensions were fit to the shared and idiosyncratic subspaces, with $K_{1}=40$. To evaluate the ability of MD2S to recover the correct number of systematic dimensions per subspace, we use three settings. First, we set $N=50$ and $K_{2}=100$ in panel (a). Moving from panel (a) to (b), we increased $N$ to 100 but kept $K_{2}$ fixed at 100. Finally, moving from panel (b) to (c), we kept $N=100$, but increase $K_{2}$ to 1,000 . For each of the above-mentioned settings, 500 permuted datasets were used to estimate the $p$-value, and 500 total simulations were run.

Across panels, if we classify values below $p=0.10$ as successful instances of uncovering signal from noise, MD2S consistently recovers the first dimension of the the shared subspace. However, if the number of observations $(N)$ is small as in panel (a), MD2S recovers the second shared dimension, which is not noise, only $17 \%$ of the time. Increasing the number of observations, as done in panel (b), improves MD2S' 

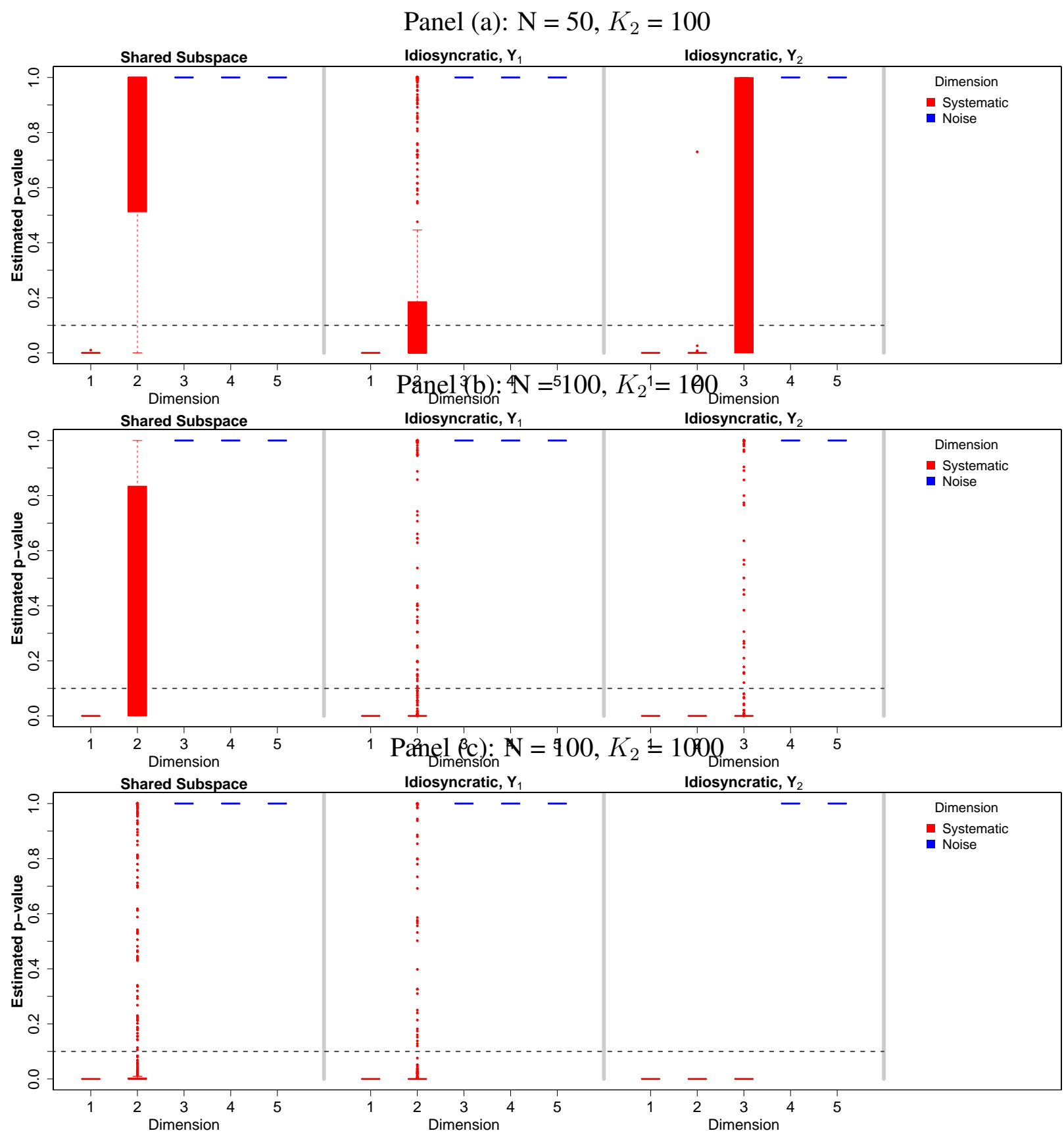

Figure 3: Estimating Dimensions. The three panels above present the results from the permutation test for the number of dimensions under three different settings. We use the same data generation process described in section 4, fixing $K_{1}=40$. For panel (a), $N=50$ and $K_{2}=100$; for panel (b), $N=100$ and $K_{2}=100$; and for panel (c), $N=100$ and $K_{2}=1000$. Values in the boxplot that fall below the dotted line at $p=0.10$ were estimated as systematic dimensions; those above were considered noise. As the three panels show, as we increase both the number of observations $N$ and the number of features $\left(K_{2}\right)$ of the larger data set, MD2S is able to detect the correct dimensionality. Note that for each of the 500 simulations per setting, 500 permuted datasets were used to recover a $p$-value. 
ability to recover the second shared dimension, as it is now detected $53 \%$ of the time. If both $N$ and $K_{2}$ are increased, as in panel (c), MD2S classifies the second shared subspace as signal $83 \%$ of the time.

A similar but less pronounced pattern, is observed for the two idiosyncratic subspaces. The noise dimensions, 3-5 in $Y_{(1)}$ and 4 and 5 in $Y_{(2)}$ are never selected. However, dimensions 2 for $Y_{(1)}$ and 3 for $Y_{(2)}$ which contain systematic information, are difficult to recover for MD2S when both $N$ and $K_{2}$ are small. In panel (a), MD2S correctly classifies dimensions 2 of $Y_{(1)}$ and 3 of $Y_{(2)}$ as signal $73 \%$ and $46 \%$ of the time, respectively. If the number of observations and features of our larger data set are increased, as in panel (c), then MD2S correctly classifies dimension 2 of $Y_{(1)}$ as signal $93 \%$ of the time, while dimension 3 of $Y_{(2)}$ is always correctly classified as signal.

\section{Combining Senate Roll Call and Text Data}

In this empirical exercise, we apply MD2S to data from speech and roll call votes in the 112th U.S. Senate. The data consist of a term document matrix of 2532 unique terms constructed from senators' floor speech found in the Congressional Record, as well as the final roll call vote matrix of 486 binary votes taken during this session. ${ }^{12}$ The data was previously analyzed using the Sparse Factor Analysis (SFA) methodology introduced in Kim, Londregan and Ratkovic (2018), who found two dimensions in the space jointly informed by words and votes. The primary dimension was qualitatively the same as the ideological dimension identified by any common scaling method applied to roll call votes from the U.S. Senate (e.g. Clinton, Jackman and Rivers, 2004, Figure 1). The second dimension was a "leadership" dimension ranging from party leaders, on one end, to rank and file members on the other.

\footnotetext{
${ }^{12}$ Speech data is collected by the Sunlight Foundation and roll call votes are obtained from VoteView. To create the document-term matrix, senators' floor speech is preprocessed following standard practices by stemming, eliminating stopwords, and analyzing all unigrams and bigrams in the text data. Infrequent terms that are not used by at least ten senators are trimmed. See Kim, Londregan and Ratkovic (2018) for a detailed discussion of the construction of the document-term matrix. Our results are robust to different trimming rules and the inclusion of bigrams (Denny and Spirling, 2018); see Supplemental Appendix B.2.2 for details.
} 
We present the results obtained from MD2S in two parts. First, we use our permutation test to assess which latent dimensions may not be noise. Second, we examine the substance of the scaled locations in the first shared subspace informed by both words and votes, as well as the first idiosyncratic dimensions specific to each type of data. We show the point estimates of the scaled locations for each senator in the results below. Results of the bootstrapped confidence intervals as described in Section 3.4 can be found in the Supplemental Appendix.

We inform the scaled locations with available senators' characteristics. ${ }^{13}$ In particular, we allow the latent variables to be a function of senators' party, gender, and seniority. We also account for measures of the number and type of committee assignments of each senator in this session. ${ }^{14}$ We include membership, which is given by the total number of committees a senator belongs to. The variable leadership is a representation of the number of committees where a senator holds a leadership position. The remaining covariates: agricultural, economics, and security, measure the proportion of committees a senator belongs to that deal with these issues. ${ }^{15}$ In the Supplemental Appendix B.2.5, we show the results from regressing the estimated locations for each subspace on this set of covariates.

Estimating dimensionality. Table 1 presents the results from the permutation test presented in section 3.4 applied to the Senate data. The table contains the results for the shared subspace and the two idiosyncratic dimensions.

Using any $p$-value threshold between 0.01 and 0.71 gives us one statistically significant dimension across the shared and idiosyncratic subspaces. In terms of explained variance, the first shared subspace explains most of the joint variance across votes and words (i.e., 96\%). For the idiosyncratic subspaces the

13 As a robustness check, in Supplemental Appendix B.2.1, we present and discuss the results without using senators' characteristics - which overall are quite similar to the results presented here.

${ }^{14}$ Senate committee assignments are obtained from Stewart and Woon (1998).

${ }^{15}$ agricultural include the committees of Agriculture, Nutrition and Forestry, Energy and Natural Resources, and Environment and Public Works. economi cs include the committees of Appropriations, Banking, Housing, and Urban Affairs, Budget, and Finance. security includes the committees of Armed Services and Homeland Security and Governmental Affairs. 


\begin{tabular}{lrrrrrr}
\hline & & \multicolumn{5}{c}{ Dimension } \\
& & $(1)$ & $(2)$ & $(3)$ & $(4)$ & $(5)$ \\
\hline Shared subspace & p-value & 0.00 & 1.00 & 1.00 & 1.00 & 1.00 \\
& $\%$ & 95.72 & 1.94 & 0.89 & 0.82 & 0.63 \\
Word subspace & p-value & 0.00 & 0.72 & 1.00 & 1.00 & 1.00 \\
& $\%$ & 40.87 & 20.43 & 15.53 & 12.13 & 11.04 \\
Vote subspace & p-value & 0.00 & 1.00 & 1.00 & 1.00 & 1.00 \\
& $\%$ & 42.54 & 19.15 & 14.84 & 13.08 & 10.40 \\
\hline
\end{tabular}

Table 1: Permutation test. \% represents the percentage of explained variance for each dimension. Using any $p$-value threshold between 0.01 and 0.71 gives us two dimensions for the shared subspace and three for the idiosyncratic word and votes subspaces.

first dimension explains $43 \%$ and $41 \%$ of the variance unique to votes and words, respectively.

Scaled locations. Since we are placing the words and the senators in the same subspace, words at one extreme are most used by legislators at the same extreme. Connecting the words with the legislators greatly aids in interpretation, as we do not only have the locations of the legislators to go by in ascertaining the substantive meaning of the dimension.

We present the scaling estimates of the first shared dimension in Figure 4. On the left panel, we present word clouds containing the top 100 positive (in red) and top 100 negative (in blue) words according to their weights in the estimated matrix of factors for the text data, $\widehat{W}_{(w o r d s)}$. The size and color intensity of each word in the wordclouds are proportional to the absolute value of the estimated weights, so words of one color are estimated as near legislators of the same color.

The right panel of figure 4 presents the estimated location of senators in the shared subspace $\hat{Z}_{s} \cdot{ }^{16}$ The shared subspace differentiates the party, placing Republicans towards the top and Democrats towards the bottom. Our estimates are highly correlated with the SFA ideal point estimates at 0.97 . With respect to scaling approaches using only data on roll call votes, our first shared dimension correlates with DW-

\footnotetext{
${ }^{16}$ Subsection B.2.4 of the Supplemental Appendix presents the estimated locations with their corresponding $95 \%$ bootstrapped percentile confidence intervals.
} 
NOMINATE (Poole, 2005) and IDEAL (Clinton, Jackman and Rivers, 2004) at 0.94 and 0.95, respectively. Therefore, the shared scale is consistent with the ideological dimension uncovered from a spatial vote choice model and from its extension to word choice. ${ }^{17}$

These correlations serve as a validation exercise, as DW-NOMINATE and IDEAL have proven to be robust methods to extract information exclusively from roll call votes. Thus, by adding words into the equation in a structured fashion, $\mathrm{MD} 2 \mathrm{~S}$ is able to recover other interesting patterns, while also recovering the expected ideological dimension from the vote data obtained by popular methods such as DW-Nominate and IDEAL. ${ }^{18}$

The words anchoring each dimension are similar to those identified in Kim, Londregan and Ratkovic (2018, see Figure 3, righthand plot). In particular, we find parliamentary control terms on the side associated with the governing Democratic majority (meet session, author meet, conduct hear) with fiscal terms on the side associated with the Republican minority, (stimulus, trillion, budget, rais tax, debt) commensurate with the party's professed fiscal concerns. If we move past the parliamentary terms, the first set of substantive terms on the Democratic side are also fiscal in nature but diametrically opposite the Republicans: wealthiest, middleclass, tax break, tax cut, and hear entitl. Therefore, by recovering the associated terms with each scaled location, we find that the first shared dimension captures well the main differences between Democrats and Republicans in terms of fiscal policy, as identified by the words most associated with each side of the scale.

In terms of idiosyncratic subspaces, we first focus our attention on the vote subspace. As illustrated by Figure 5, we find a significant first dimension that organizes voting, but is unrelated to floor speech. On one extreme, this dimension is anchored by fiscal conservative senators DeMint, Lee, Toomey, Paul,

\footnotetext{
${ }^{17}$ The shared scale recovered by V-BIFBA applied to the Senate data correlates with our shared scale at 0.89 . The correlation of V-BIFBA with SFA, DW-Nominate and IDEAL is $0.91,0.91$ and 0.95 , respectively.

${ }^{18}$ See Supplemental Appendix B.2.3 for the overall correlations, within-party correlations, and different scatter plots showing the similarities across different scaling methods.
} 

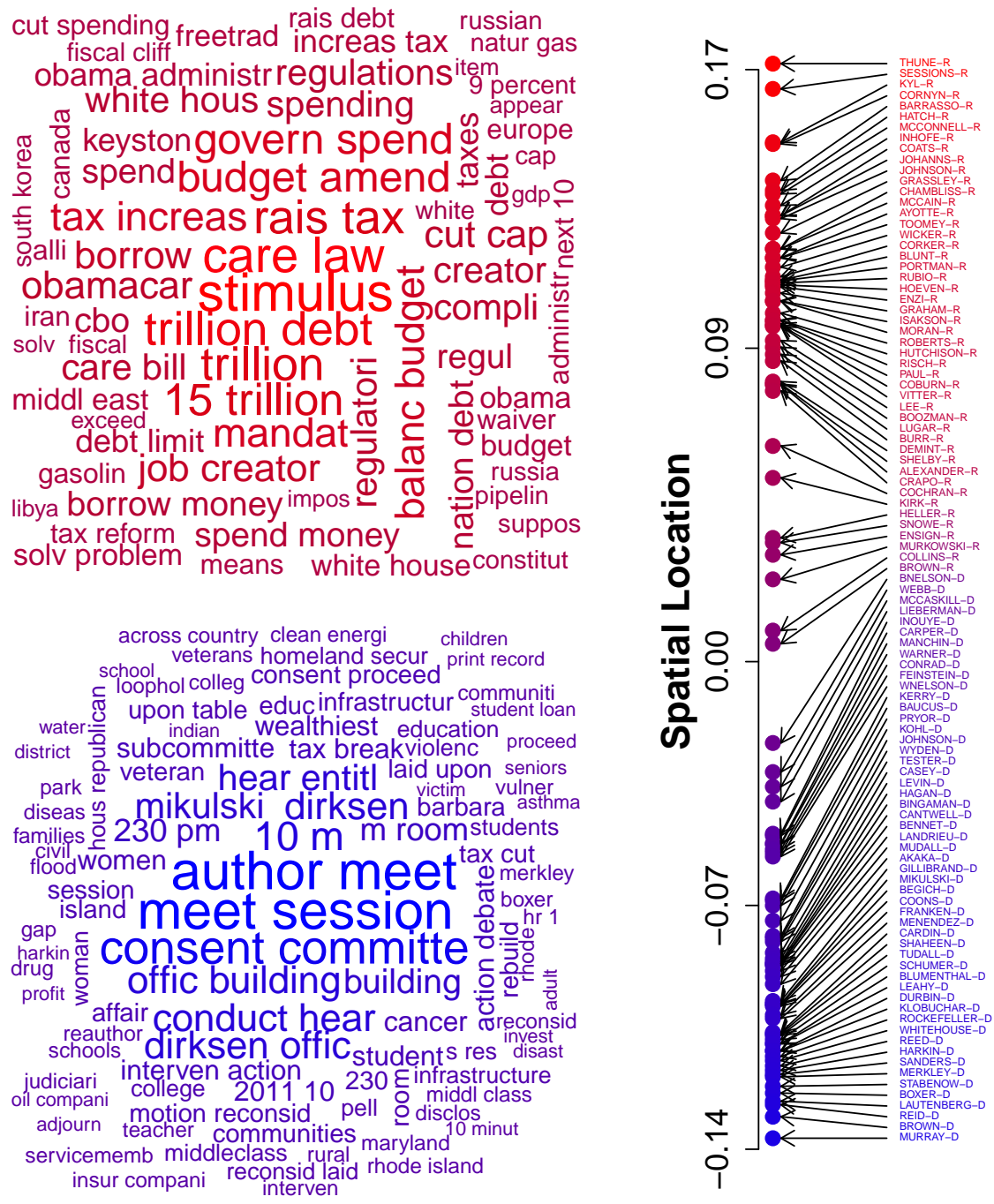

Figure 4: Shared Subspace Locations Estimated via MD2S for the Members of the 112th U.S. Senate.

and Risch, noted Tea Party and small government enthusiasts. In terms of the covariates included in the estimation, senators assigned to a leadership position in committees related to agricultural and economic issues are significantly correlated with this extreme of the scale. The other extreme of the vote subspace is anchored by prominent and more moderate senior senators like Schumer, Boxer, and Collins, who have been reelected at least once and hold a leadership position in a Senate committee. As shown in the appendix, we find that seniority and more leadership assignments, as well as membership in committees focused on national security issues, are systematically associated with positive locations 
in the vote subspace.

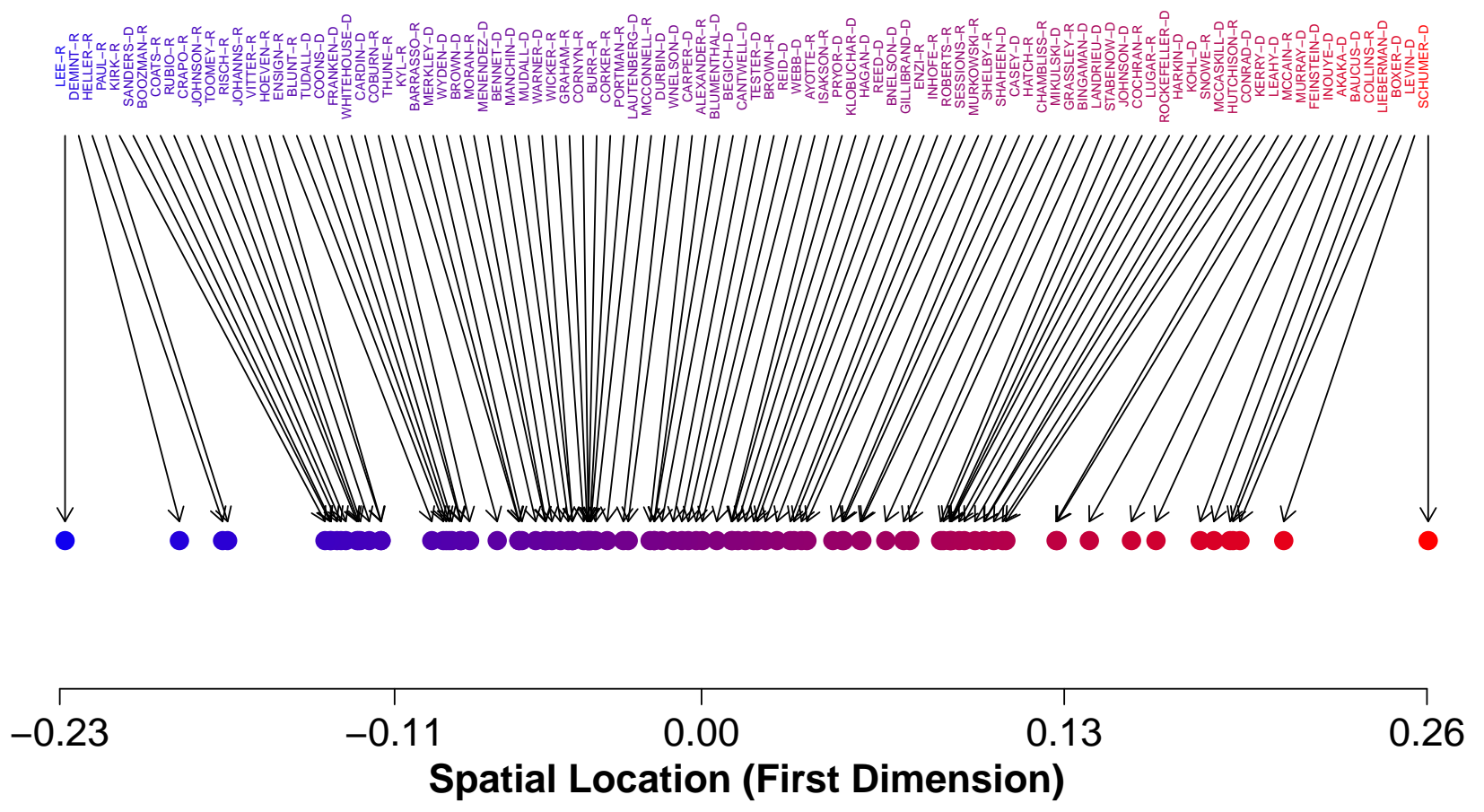

Figure 5: Idiosyncratic Vote Subspace Locations Estimated via MD2S for the Members of the 112th U.S. Senate. 
paid done deal oil democrat kind gas balanc budget say go ohuge bad incom gone chart littl bit \% anybodi kidvote wealth entith bigright now o $\overline{\bar{\delta}}$ guess Someththink medicar \& littl $\bar{\sigma}^{\text {T. }}$ tell republican taxes hitlook anyth cot $_{\text {sit }}$ talk lotsee tax know - money thing get said sobs Othings everybodi letwant ơ els go get cut happen pretti $\frac{\pi}{0}$ w ggo backtrillion medicare coliff wrong social secur whole stolk side evensocial security one thing fine wors can getpay saying job somebodi

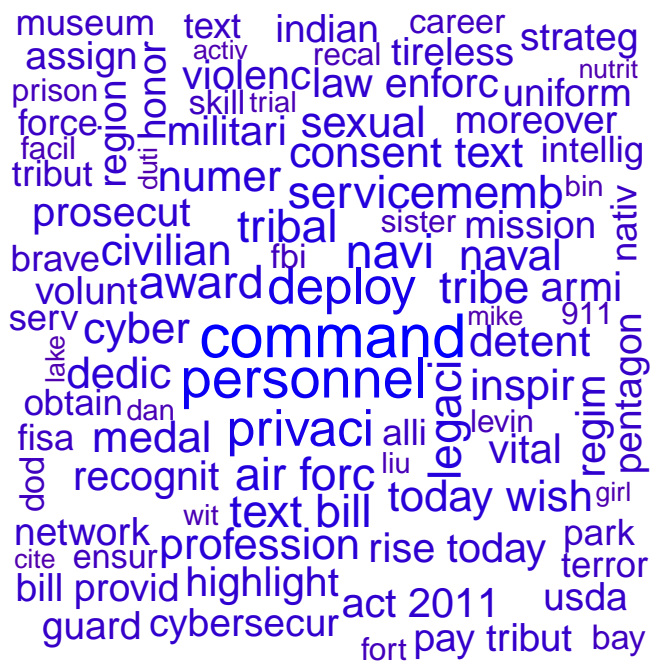

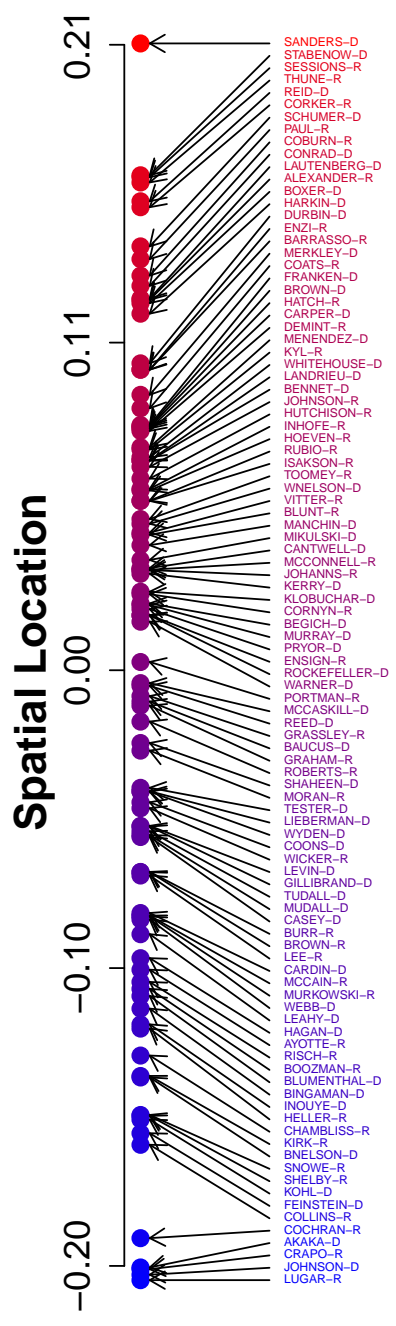

Figure 6: Idiosyncratic Word Subspace Locations Estimated via MD2S for the Members of the 112th U.S. Senate. First Dimension.

Similar to the results for the shared subspace, Figure 6 shows senators' locations in the first dimension of the word subspace along with the word clouds of the top 100 words associated with each side of the scale given by the estimated text factor $\widehat{B}_{(\text {words })}$. In terms of the scaled locations, we have on one end, senators who put emphasis on national security issues, such as prominent members of the Committees on Armed Services and Homeland Security, as well as Governmental Affairs like senators Johnson, Akaka, and Collins. The associated terms on this extreme relate to the military (e.g, command, deploy, navi, air 
forc), as well as personnel and privacy-protection issues (e.g., privaci, personnel, civilian). The opposite side of the words subspace is anchored by senators of both parties (Sanders, Stabenow, Sessions and Thune) addressing budget issues, with associated terms such as tax, deficit, debt, money and medicare.

Notice that the estimated locations in these idiosyncratic vote and words dimensions allows us to further differentiate senators found to be moderate in the shared dimension, but that have substantive differences specific to either their roll-call or floor speech behavior.

\section{Conclusion}

As we enter a period of "big data," we encourage political scientists to think not just of analyzing large datasets but also how to combine data from disparate sources. We present such a method here, for scaling data from two separate datasets. The method, MD2S, successfully incorporates information from two different data sources, generating scaled locations with a higher internal validity than analyzing the two datasets separately. We include methods for checking validity, separating systematic dimensions from noise, and a way to relate scaled locations to covariates, all fit using an efficient statistical algorithm.

The method also allows the user to use the scaled locations from both datasets to help infer the meaning of the latent dimensions. In our empirical application, scaled locations were also associated with words that let us better interpret the meaning of the estimated latent scales. The idiosyncratic subspaces also offers new insights, allowing us to identify dimensions in which the members at the extremes of the shared subspace differed.

We anticipate several ways in which this project can be moved forward. First, we have presented the method in a geometric, least squares framework. Placing the method in a probabilistic framework will allow for an extension to commonly used Bayesian techniques (Hare et al., 2015; Tipping and Bishop, 1999). We also plan to extend the method to allow for cross-time comparisons, so as to place multiple 
observations in the same space over time. As noted above, an important avenue of future research is to scale information coming from more than two datasets e.g., scaling Senators' roll calls, floor speeches, and social media statements. In principle, our framework and identification assumptions can be generalized to multiple databases. We leave for future research an empirical implementation of this extension to the method.

\section{Appendix}

\section{A Proof of Proposition 1}

We first derive score conditions for the IBFA, extending the model of Tipping and Bishop (1999). We then implement a Minorize-Maximization (MM) algorithm for estimation (for the use of this class of algorithm in scaling, see Borg and Groenen, 2005). The estimation procedure works by deriving a minorizing function that lies weakly below the true objective, maximizes, and iterates to convergence. ${ }^{19}$

The model and likelihood. This section follows Tipping and Bishop (1999). The two data sets, $Y_{(1)}$ and $Y_{(2)}$ are modeled in terms of a shared subspace $Z_{S}$ as well as dataset specific subspaces, $Z_{(1)}$ and $Z_{(2)}$, such that for column $j$ :

$$
Y_{(m) j} \mid W_{(m) j}, B_{(m) j}, L_{(m)}, D_{(m)} \sim \mathcal{N}\left(Z_{S} L_{(m)} W_{(m) j}+Z_{(m)} D_{(m)} B_{(m) j}, \sigma_{(m)}^{2} I_{N}\right)
$$

for $m \in\{1,2\}$. Marginalizing over $W_{(m)}$ and $B_{(m)}$ gives the unconditional densities for $Y_{(m) j}$ as

$$
Y_{(m) j} \sim \mathcal{N}\left(0_{N}, C_{m}\right)
$$

\footnotetext{
${ }^{19}$ The $Q$ function in the popular EM algorithm is a minorizing function.
} 
where $C_{m}=Z_{S} L_{(m)}^{2} Z_{S}^{\top}+Z_{(m)} D_{(m)}^{2} Z_{(m)}^{\top}+\sigma_{1}^{2} I_{N}$

The log-likelihood of $\left(Z_{S}, Z_{(1)}, Z_{(2)}, L_{(1)}, L_{(2)}, D_{(1)}, D_{(2)}\right)$ is then

$$
\begin{aligned}
& l\left(Z_{S}, Z_{(1)}, Z_{(2)}, L_{(1)}, L_{(2)}, D_{(1)}, D_{(2)} \mid Y_{(1)}, Y_{(2)}\right)= \\
& -\frac{1}{2}\left\{N\left(K_{1}+K_{2}\right) \log (2 \pi)-K_{1} \log \left(\left|C_{1}\right|\right)-K_{2} \log \left(\left|C_{2}\right|\right)-\operatorname{tr}\left(Y_{(1)} Y_{(1)}^{\top} C_{1}^{-1}+Y_{(2)} Y_{(2)}^{\top} C_{2}^{-1}\right)\right\} .
\end{aligned}
$$

Denoting $L_{(2)}^{2}=\lambda^{2} L_{(1)}^{2}$, the score conditions for the shared subspace model are

$$
\begin{aligned}
\frac{\partial l(\cdot)}{\partial Z_{S}} & =\left\{\frac{1}{2}\left(K_{1} C_{1}^{-1}+K_{2} C_{2}^{-1}\right)-\frac{1}{2}\left\{C_{1}^{-1} Y_{(1)} Y_{(1)}^{\top} C_{1}^{-1}+\lambda^{2} C_{2}^{-1} Y_{(2)} Y_{(2)}^{\top} C_{2}^{-1}\right\}\right\} Z_{S} L_{(1)}^{2} \\
\frac{\partial l(\cdot)}{\partial Z_{(m)}} & =\left\{\frac{1}{2} K_{m} C_{m}^{-1}-\frac{1}{2} C_{m}^{-1} Y_{(m)} Y_{(m)}^{\top} C_{m}^{-1}\right\} Z_{(m)} D_{(m)}^{2}
\end{aligned}
$$

It may appear at first that $Z_{(m)}$ and $Z_{S}$ are solutions to an eigen problem of the form $A Z=\lambda Z$. This does not immediately follow from Equations (23) - (24), though, because $Z_{(m)}$ and $Z_{(S)}$ enter into $C_{m}$ nonlinearly. The work in the proof below comes from using the identification conditions (equations 6 to 10) and making use of the Woodbury identity to isolate $Z_{S}$ in $C_{m}^{-1}$. With this done, it is apparent that the maximum likelihood estimates are indeed singular vectors. We formalize that result in the Proposition 1, which is given in the text.

\section{Proof of Proposition 1.}

1. We proceed in two steps. First, we simplify the term $C_{m}^{-1} Z_{(m)} D_{(m)}^{2}$, leaving it a function of only $Z_{(m)}$ and not $Z_{S}$. Second, we substitute this simplified term back into the score conditions, showing that $Z_{(m)}$ are singular vectors. 
First, denote $A=\left(Z_{m} D_{(m)}^{2} Z_{(m)}^{\top}+\sigma_{(m)}^{2} I_{n}\right)$ and $U=\left(L_{(m)}^{-2}+Z_{S}^{\top} A^{-1} Z_{S}\right)$. Then,

$$
\begin{array}{rlrl}
C_{m}^{-1} Z_{(m)} D_{(m)}^{2} & =C_{m}^{-1} M\left(Z_{S}\right) Z_{(m)} D_{(m)}^{2} & & Z_{S} \perp Z_{(m)} \\
& =\left\{A^{-1}-A^{-1} Z_{S} U^{-1} Z_{S}^{\top} A^{-1}\right\} M\left(Z_{S}\right) Z_{(m)} D_{(m)}^{2} & & \text { Woodbury identity to } C_{m}^{-1} \\
& =\left(Z_{(m)} D_{(m)}^{2} Z_{(m)}^{\top}+\sigma_{(m)}^{2} I_{N}\right)^{-1} M\left(Z_{S}\right) Z_{(m)} D_{(m)}^{2} &
\end{array}
$$

where the last line follows from distributing and that $A^{-1}$ is not a function of $Z_{S}$, leaving the second summand linear in $Z_{S}$ and therefore annihiliated by $M\left(Z_{S}\right)$. We further simplify through reapplying the Woodbury identity to $\left(Z_{(m)} D_{(m)}^{2} Z_{(m)}^{\top}+\sigma_{(m)}^{2} I_{N}\right)^{-1}$ :

$$
\begin{aligned}
& =\frac{1}{\sigma_{(m)}^{2}}\left\{I_{N}-Z_{(m)}\left(\sigma_{(m)}^{2} D_{(m)}^{-2}+Z_{(m)}^{\top} Z_{(m)}\right)^{-1} Z_{(m)}^{\top}\right\} M\left(Z_{S}\right) Z_{(m)} D_{(m)}^{2} \\
& =M\left(Z_{S}\right) Z_{(m)} \widetilde{D}_{(m)}
\end{aligned}
$$

where we denote the diagonal matrix $\widetilde{D}_{(m)}=\frac{1}{\sigma_{(m)}^{2}}\left\{I_{N}-\left(\sigma_{(m)}^{2} D_{(m)}^{-2}+I_{Q_{(m)}}\right)^{-1}\right\}$. That this matrix is diagonal is crucial to our result, illustrating where the advantage of the PPCA enters our results.

Substituting into the score conditions gives:

$$
\begin{array}{lr}
K_{m} C_{m}^{-1} Z_{(m)}-\frac{1}{\sigma_{(m)}^{2}} C_{m}^{-1} Y_{(m)} Y_{(m)}^{\top} M\left(Z_{S}\right) Z_{(m)} \widetilde{D}_{m}=0_{N \times Q_{m}} & \\
\Rightarrow K_{m} Z_{(m)}-\frac{1}{\sigma_{(m)}^{2}} Y_{(m)} Y_{(m)}^{\top} M\left(Z_{S}\right) Z_{(m)} \widetilde{D}_{m}=0_{N \times Q_{m}} & \text { Pre-multiply } C_{m} \\
\Rightarrow K_{m} M\left(Z_{S}\right) Z_{(m)}-\frac{1}{\sigma_{(m)}^{2}} M\left(Z_{S}\right) Y_{(m)} Y_{(m)}^{\top} M\left(Z_{S}\right) Z_{(m)} \widetilde{D}_{m}=0_{N \times Q_{m}} & \text { Pre-multiply } M\left(Z_{S}\right) \\
\Rightarrow K_{m} Z_{(m)}-\frac{1}{\sigma_{(m)}^{2}} M\left(Z_{S}\right) Y_{(m)} Y_{(m)}^{\top} M\left(Z_{S}\right) Z_{(m)} \widetilde{D}_{m}=0_{N \times Q_{m}} & M\left(Z_{S}\right) Z_{(m)}=Z_{(m)} \\
\Rightarrow K_{m} Z_{(m)} I_{L_{m}}-\frac{1}{\sigma_{(m)}^{2}} W_{(m)} Z_{(m)} \widetilde{D}_{m}=0_{N \times Q_{m}}, &
\end{array}
$$


where we define $W_{(m)} \equiv M\left(Z_{S}\right) Y_{(m)} Y_{(m)}^{\top} M\left(Z_{S}\right)$ in the last line. Considering this last equality columnwise shows that each column of $Z_{(m)}$ is a singular vector of $W_{(m)}$, which was to be shown.

2. Denote $Z_{S \mid m}$ as the first $L_{S}$ principal components of $Y_{(m)}^{\top} M\left(Z_{(m)}\right)$. To prove part (a), just repeat the proof for point 1 using $M\left(Z_{(1)}\right)$ and $M\left(Z_{(2)}\right)$ in equation (23). Then, by a similar argument, the maximum likelihood estimates of $Z_{S}$ are proportional to singular vectors of a weighted average of $Y_{(1)} Y_{(1)}^{\top}$ and $Y_{(2)} Y_{(2)}^{\top}$. To prove part (b), we maximize a minorizing function that lies weakly below the true likelihood function. To generate the minorizing function, note

$$
\begin{aligned}
& Y_{(m)}^{\top} Y_{(m)} \geqslant \mathbb{E}\left(Y_{(m)}^{\top} Y_{(m)} \mid Z_{S}, Z_{(m)}, \sigma_{(m)}^{2}\right)=C_{(m)} \\
\Rightarrow & \left(Y_{(m)}^{\top} Y_{(m)}\right)^{-1} \leqslant C_{(m)}^{-1}
\end{aligned}
$$

with the inequalities meant in a matrix sense. Define

$$
\begin{aligned}
& Q\left(Z_{S}, Z_{(1)}, Z_{(2)} \mid Y_{(1)}, Y_{(2)}, C_{(1)}^{-1}, C_{(2)}^{-1}\right)= \\
& -\frac{1}{2}\left\{N\left(K_{1}+K_{2}\right) \log (2 \pi)+K_{1} \log \left(\left|C_{1}\right|\right)+K_{2} \log \left(\left|C_{2}\right|\right)\right. \\
& \left.\quad+\operatorname{tr}\left(C_{2}^{-1} Y_{(2)} Y_{(2)}^{\top} Y_{(1)} Y_{(1)}^{\top} C_{1}^{-1}+C_{1}^{-1} Y_{(1)} Y_{(1)}^{\top} Y_{(2)} Y_{(2)}^{\top} C_{2}^{-1}\right)\right\}
\end{aligned}
$$

By construction,

$$
Q\left(Z_{S}, Z_{(1)}, Z_{(2)} \mid Y_{(1)}, Y_{(2)}, C_{(1)}^{-1}, C_{(2)}^{-1}\right) \leqslant l\left(Z_{S}, Z_{(1)}, Z_{(2)} \mid Y_{(1)}, Y_{(2)}\right)
$$

Following the steps in the proof of part (1), $Z_{S}$ is clearly proportional to a left singular vector of a weighted average of $A$ and $A^{\top}$, where $A=Y_{(2)} Y_{(2)}^{\top} Y_{(1)} Y_{(1)}^{\top}$. That the maximizer of the minorizing 
function at convergence is also the ML estimate follows from invariance of the ML estimator. 


\section{References}

Albert, James H. and Siddhartha Chib. 1993. "Bayesian Analysis of Binary and Polychotomous Response Data." Journal of the American Statistical Association 88(422):669-679.

Aldrich, John and Richard McKelvey. 1977. "A Method of Scaling with Applications to the 1968 and 1972 Presidential Elections.” American Political Science Review 71(1):111-130.

Anderson, T.W. 1989. "Linear Latent Variable Models and Covariance Structures.” Journal of Econometrics 41:91-119.

Bach, Francis and Michael Jordan. 2005. A Probabilistic Interpretation of Canonical Correlation Analysis. Technical Report 688 Department of Statistics, University of California at Berkeley.

Bafumi, Joseph and Michael Herron. 2010. "Leapfrog Representation and Extremism: A Study of American Voters and Their Members in Congress.” American Political Science Review 104(3):519-542.

Barbera, Pablo. 2016. "Birds of the Same Feather Tweet Together. Bayesian Ideal Point Estimation Using Twitter Data." Political Analysis 23(1):76-91.

Bonica, Adam. 2014. "Mapping the Ideological Marketplace." American Journal of Political Science 58(2):367-386.

Borg, Ingwer, Groenen, Patrick J.F. and Patrick Mair. 2013. Applied Multidimensional Scaling. Springer.

Borg, Ingwer and Patrick J.F. Groenen. 2005. Modern Multidimensional Scaling:Theory and Applications. Springer.

Browne, M. W. 1979. “The Maximum-likelihood Solution in Inter-battery Factor Analysis.” British Journal of Mathematical and Statistical Psychology 32:75-86. 
Clinton, Joshua, Simon Jackman and Doughlas Rivers. 2004. "The Statistical Analysis of Roll Call Data." American Political Science Review 98(2):355-370.

Coppedge, Michael, John Gerring, Staffan I. Lindberg, Svend-Erik Skaaning, Jan Teorell, with David Altman, Michael Bernhard, M. Steven Fish, Adam Glynn, Allen Hicken, Carl Henrik Knutsen, Kelly McMann, Pamela Paxton, Daniel Pemstein, Jeffrey Staton, Brigitte Zimmerman, Rachel Sigman, Frida Andersson, Valeriya Mechkova and Farhad Miri. 2015. "V-Dem Codebook v5." Varieties of Democracy (V- Dem) Project.

Denny, Matthew J. and Arthur Spirling. 2018. “Text Preprocessing For Unsupervised Learning: Why It Matters, When It Misleads, And What To Do About It.” Political Analysis 26(2):168-189.

Gentzkow, Matthew and Jesse M Shapiro. 2010. "What drives media slant? Evidence from US daily newspapers." Econometrica 78(1):35-71.

Goplerud, Max. 2019. “A Multinomial Framework for Ideal Point Estimation.” Political Analysis 27(1):69-89.

Groseclose, Tim and Jeffrey Milyo. 2005. “A measure of media bias.” The Quarterly Journal of Economics 120(4):1191-1237.

Gupta, Sunil Kumar, Dinh Phung, Brett Adams and Svetha Venkatesh. 2011. Advances in Knowledge Discovery and Data Mining. Lecture Notes in Computer Science Springer-Verlag chapter "A Bayesian Framework for Learning Shared and Individual Subspaces from Multiple Data Sources”, pp. 136-147.

Hahn, P. Richard, Carlos M. Carvalho and James G. Scott. 2012. “A Sparse factor Analytic Probit Model for Congressional Voting Patterns." Journal of the Royal Statistical Society, Series A 61(4):619-635. 
Hansen, Stephen, Michael McMahon and Andrea Prat. 2018. "Transparency and Deliberation within the FOMC: A Computational Linguistics Approach.” Quarterly Journal of Economics 133(2):801-870.

Hare, Christopher, David A. Armstrong II, Ryan Bakker Royce Carroll and Keith T. Poole. 2015. "Using Bayesian Aldrich-McKelvey Scaling to Study Citizens’ Ideological Preferences and Perceptions.” American Journal of Political Science 59(3):759-774.

Hastie, Trevor, Robert Tibshirani and Jerome Friedman. 2013. The Elements of Statistical Learning. 10 ed. New York: Springer-Verlag.

Hobbs, William. 2017. “Pivoted Text Scaling for Open-Ended Survey Responses.” Unpublished manuscript.

Hobbs, William R and Margaret E Roberts. 2018. "How sudden censorship can increase access to information." American Political Science Review 112(3):621-636.

Hoff, Peter D. 2007. "Extending the Rank Likelihood for Semiparametric Copula Estimation.” The Annals of Applied Statistics 1(1):265-283.

Jackman, Simon and Shawn Trier. 2008. “Democracy as a Latent Variable.” American Journal of Political Science 52(1):201-17.

Jacoby, William G. 1986. "Levels of conceptualization and reliance on the liberal-conservative continuum." The Journal of Politics 48(2):423-432.

Jacoby, William G. 2009. "Public opinion during a presidential campaign: Distinguishing the effects of environmental evolution and attitude change.” Electoral Studies 28(3):422-436.

Jacoby, William G. and David A. Armstrong II. 2014. "Bootstrap Confidence Regions for Multidimensional Scaling Solutions.” American Journal of Political Science 58(1):264-278. 
Jessee, Stephen. 2016. “(How) Can We Estimate the Ideology of Citizens and Political Elites on the Same Scale?" American Journal of Political Science 60(4):1108-1124.

Keele, Luke, Corrine McConnaughy and Ismail White. 2012. "Strengthening the Experimenter's Toolbox: Statistical Estimation of Internal Validity.” American Journal of Political Science 56(2):484-499.

Kellerman, Michael. 2012. "Estimating Ideal Points in the British House of Commoms Using Early Day Motions.” American Journal of Political Science 56(3):757-771.

Kim, In Song, John Londregan and Marc Ratkovic. 2018. "Estimating Spatial Preferences from Votes and Text." Political Analysis. 26(2):210-229.

Klami, Arto, Seppo Virtanen and Samuel Kaski. 2013. “Bayesian Canonical Correlation Analysis.” Journal of Machine Learning Research 14(Apr):965-1003.

Ladha, Krishna. 1991. “A Spatial Model of Leglslative Voting with Perceptual Error.” Public Choice 68(1/3):151-74.

Lauderdale, Benjamin and Tom Clark. 2014. "Scaling Politically Meaningful Dimensions Using Texts and Votes." American Journal of Political Science 58(3):754-771.

Lewis, Jeffrey B. and Chris Tausanovitch. 2015. When Does Joint Scaling Allow For Direct Comparisons of Preferences? Technical report University of California, Los Angeles.

Mair, Patrick, Ingwer Borg and Thomas Rusch. 2016. “Goodness-of-Fit Assessment in Multidimensional Scaling and Unfolding.” Multivariate Behavioral Research 51(6):772-789.

Martin, Gregory J and Ali Yurukoglu. 2017. "Bias in cable news: Persuasion and polarization.” American Economic Review 107(9):2565-99. 
Murphy, Kevin P. 2012. Machine Learning: A Probabilistic Perspective. MIT press.

Murray, Jared S., David B. Dunson, Lawrence Carin and Joseph E. Lucas. 2013. “Bayesian Gaussian Copula Factor Models for Mixed Data.” Journal of the American Statistical Association 108(502):656665.

Poole, Keith and Howard Rosenthal. 1997. Congress: A Political Economic History of Roll Call Voting. New York: Oxford University Press.

Poole, Keith T. 2005. Spatial Models of Parliamentary Voting. Analytical Methods for Social Research Cambridge: Cambridge University Press.

Quinn, Kevin M. 2004. “Bayesian Factor Analysis for Mixed Ordinal and Continuous Responses.” Political Analysis 12(4):338-353.

Roberts, Molly, Brandon Stewart, Dustin Tingley, Christopher Lucas, Jetson Leder-Luis, Shana Gadarian, Bethany Albertson and David Rand. 2014. "Structural Topic Models for Open Ended Survey Responses.” American Journal of Political Science 58(4):1064-1082.

Rockova, V. and E. I. George. 2016. "Fast Bayesian factor analysis via Automatic Rotations to Sparsity." Journal of the American Statistical Association 111(516):1608-1622.

Shor, Boris and Nolan McCarty. 2011. "The Ideological Mapping of American Legislatures.” American Political Science Review 105(03):530-551.

Stewart, Charles III and Jonathan Woon. 1998. "Congressional Committee Assignments, 103rd to 114th Congresses, 1993-2017:Senate, 11/17/2017.”.

Tausanovitch, Chris and Christopher Warshaw. 2013. "Measuring Constituent Policy Preferences in Congress, State Legislatures, and Cities.” The Journal of Politics 75(02):330-342. 
Tipping, Michael E. and Christopher M. Bishop. 1999. "Probabilistic Prncipal Component Analysis." Journal of the Royal Statistcal Society, Series B 61(3):611-622.

Tucker, Ledyard R. 1958. “An Inter-Battery Method of Factor Analysis.” Psychometrika 23(2):111-136.
Abstract: 163 Words
Body of Paper: 7378 Words 